\title{
Acquisition of an olfactory associative task triggers a regionalized down-regulation of adult born neuron cell death
}

\section{Sébastien Sultan, Julie M. Lefort, Joëlle Sacquet, Nathalie Mandairon and Anne Didier*}

CNRS, UMR5020; INSERM, U1028; Lyon Neuroscience Research Center, Neuroplasticity and Neuropathology of olfactory perception Team, University of Lyon, Lyon; University Lyon1, Villeurbanne, France

\section{Edited by:}

Silvia De Marchis, University of Turin, Italy

\section{Reviewed by:}

Armen Saghatelyan, Université Laval,

Canada

Isabelle Caillé, CNRS, France

\section{${ }^{*}$ Correspondence:}

Anne Didier, Lyon Neuroscience

Research Center, Université Lyon 1,

50 Avenue Tony Garnier, 69007 Lyon,

France.

e-mail: didier@olfac.univ-lyon1.fr
Associative olfactory learning increased survival of adult born granule interneurons in the olfactory bulb (OB) at regions which are specific to the learned odorant. However, the mechanism shaping this odor-specific distribution of newborn neurons and its temporal relationship with the learning process are unknown. In the present study, using Bromodeoxyuridine or activated-caspase3 labeling, newborn and apoptotic cells respectively were mapped in the granule cell layer (GCL) of the $\mathrm{OB}$, just before, during, and at the end of odor conditioning or pseudo-conditioning in adult mice. Results indicate that before and during training, when the task is not yet acquired, conditioned and pseudo-conditioned animals displayed the same density of newborn neurons. However, at the end of the conditioning, when the animals mastered the task, the density of newborn cells remained elevated in conditioned animals while it decreased in pseudo-conditioned animals suggesting newborn cell death in that group. Indeed, using Activated-Caspase3/BrdU co-labeling, we found that the proportion of newborn cells among dying cells was reduced in conditioned animals mastering the task compared to non-expert conditioned or pseudoconditioned animals. The overall level of cell death did not change across training and was similar in conditioned and pseudo-conditioned groups, indicating that BrdU-positive cells were spared to the detriment of non-labeled cells. In addition, a fine analysis of cell distribution showed an uneven distribution of apoptotic cells, with lower densities in the medial part of the GCL where the density of newborn cells is high in conditioned animals. We conclude that acquisition of the task triggered the rescue of newborn neurons by a targeted regulation of cell death.

Keywords: adult neurogenesis, olfactory bulb, cell death, learning, mice, BrdU, behavior

\section{INTRODUCTION}

New neurons are provided throughout life to the olfactory bulb (OB), the first central relay of olfactory information processing. These adult born neurons originate from stem cells proliferating in the subventricular zone of the lateral ventricles, giving birth to neuroblasts which then migrate to the $\mathrm{OB}$. Within the $\mathrm{OB}$, neuroblasts differentiate into inhibitory granules cells (GCs) for the majority of them and to a lesser extent into periglomerular cells, and integrate the bulbar circuit (Lledo et al., 2006). Dendritic and synaptic development of newborn cells take about 1 month, a critical period during which they are very sensitive to olfactory experience for their morphological (Kelsch et al., 2009; Livneh et al., 2009), neurochemical development (Bovetti et al., 2009), and their long-term survival (Petreanu and Alvarez-Buylla, 2002; Winner et al., 2002; Yamaguchi and Mori, 2005; Mandairon et al., 2006b; Mouret et al., 2008).

The $\mathrm{OB}$ is heavily involved in odor learning and memorization. For instances, electrophysiological recordings have shown that the response of mitral cells, the relay cells of the OB, are modified when the odor is coupled to a positive or negative reinforcement (Kay and Laurent, 1999) and bulbar network oscillations evolve with expertise in the task (Martin et al., 2004). Immediate early gene mapping also revealed cellular plasticity in the OB following learning (Salcedo et al., 2005; Mandairon et al., 2008; Busto et al., 2009; Moreno et al., 2009). All together these data indicate that the bulbar network undergoes experiencedependent plasticity. Inhibitory interneurons which interact through dendro-dendritic synapses with the mitral cells to shape the output message of the OB to higher olfactory centers, are involved in experience-dependent plasticity of the $\mathrm{OB}$ network (Shepherd et al., 2007).

Numerous studies over the last years have linked olfactory learning and memory to modulation of adult born GCs of the OB (Rochefort et al., 2002; Alonso et al., 2006; Mandairon et al., 2006a; Mouret et al., 2008; Lazarini et al., 2009; Moreno et al., 2009; Valley et al., 2009; Veyrac et al., 2009; Kermen et al., 2010; Sultan et al., 2010). In these studies, the number of newborn GCs was increased after olfactory enrichment or learning, suggesting that learning rescued some newborn neurons from the death which normally occurs during the first weeks after cell birth (Petreanu and Alvarez-Buylla, 2002; Winner et al., 2002; Mandairon et al., 2006b). These findings, together with the ability of newborn GCs to display long-term potentiation (Nissant et al., 2009) set the basis for the hypothesis that adult born cells could be important for olfactory learning or memory.

Recently, we have characterized further the role of adult born GCs in a paradigm of associative olfactory learning (Kermen et al., 2010; Sultan et al., 2010). We have shown that more adult born GCs survived after learning in odor-specific areas of the granule cell layer (GCL) and are required for long-term memory of the 
task. However, the exact temporal relationship between survival of newborn cells and the learning process as well as the mechanism by which they are grouped in odor-specific areas are unknown.

To address this issue, we used cellular mapping to analyze the number, the spatial distribution, and the death of newborn cells in the GCL of the OB before, during, and after olfactory conditioning. Results reveal that the patterned distribution of adult born cells in the GCL after learning is due to a region-specific reduction in newborn cell death which correlates tightly with the time point at which animals have acquired the task.

\section{MATERIALS AND METHODS ANIMALS}

Thirty-four male C57Black6/J mice (Charles River, L'Arbresles, France) aged 8 weeks at the beginning of the experiments were used. All mice were housed under a 12-h light/dark cycle in an environmentally controlled room. Mice had free access to water and food except during the olfactory learning period (see below). All behavioral training was conducted in the afternoon (14:00-17:00). Every effort was made to minimize both the number of animals used and their suffering during the experimental procedure in accordance with the European Community Council Directive of November 24, 1986 (86/609/EEC) and the University Lyon1 Ethical Committee.

\section{EXPERIMENTAL SCHEME}

Animals were injected with the cell division marker 5-Bromo-2'deoxyuridine (BrdU) 13 days before the beginning of training, to allow newborn cells to migrate to the $\mathrm{OB}$ and to be in their critical period of development during training (Mandairon et al., 2006b; Mouret et al., 2008; Kelsch et al., 2009). Three days before the onset of conditioning, all the animals were submitted to a 3-day pre-training followed on D0 by a 5-day olfactory conditioning ( $n=17)$ or pseudo conditioning $(n=15$; see below). A retention test was performed 5 days after the end of conditioning. Animals were sacrificed at different time points: before conditioning (pretraining; $n=5$ ), after 3 days of training ( $n=5$ conditioned and $n=5$ pseudo-conditioned animals), after the complete conditioning at day 5 ( $n=5$ conditioned and $n=5$ pseudo-conditioned animals) and 5-day post training ( $n=7$ conditioned and $n=5$ pseudoconditioned animals). A total of seven experimental groups were thus studied (Figure 1A). After behavioral testing, animals were sacrificed and histological techniques were applied to measure neurogenesis, neuronal differentiation, and cell death in the GCL.

\section{BEHAVIORAL EXPERIMENTS Experimental set up}

All mice were tested on a computer-assisted 2-hole board apparatus $(40 \mathrm{~cm} \times 40 \mathrm{~cm})$ run by specific software (Mandairon et al., 2009). The trial started by placing the mouse on the board, and the sequence and duration of nose poking into the holes $(3 \mathrm{~cm}$ diameter, $4.5 \mathrm{~cm}$ deep) were automatically recorded. A polypropylene swab was placed at the bottom of the hole, covered with bedding. For trials involving odors, the swab was impregnated with $20 \mu \mathrm{L}$ of pure odorant. The bedding was replaced after every trial. Between each trial, mice were put back in their home cage.

\section{Olfactory associative learning \\ Odorants}

+Limonene (Purity > 97\%, Sigma-Aldrich, Saint Louis, MO, USA)

was used in this experiment.

\section{Pre-training}

Mice were first trained to retrieve a reward (small bit of sweetened cereal, Kellogg's, Battle Creek, MI, USA) by digging through the bedding while no odor was present. The mouse was put in the start area and was allowed to dig for $2 \mathrm{~min}$. During the first few trials the reward was placed on the top of the bedding of one of the holes. After several successful retrievals, the reward was buried deeper into the bedding. Pre-training was considered to be complete when a mouse could successfully retrieve a reward that was deeply buried in the bedding (from 8 to 12 trials).

\section{Conditioning}

During the olfactory learning experiments, water was continuously available, but the mice were food-deprived for 5 days before pretraining, to obtain a $5-10 \%$ reduction in body weight. Conditioning consisted of five sessions (one per day) of four trials (2 min per trial, inter trial interval of $15 \mathrm{~min}$ ). For each trial, the mice were placed on the board and a reward (Kellogs's cereal) systematically associated with the odorant, was randomly placed in one of the two holes to avoid spatial learning. The other hole was not odorized and did not contain any reward. In the pseudo-conditioned groups, the reinforcement was randomly associated with either the odorized hole or the non-odorized hole. For each trial, the percentage of correct choice (first nose poke in the odorized hole) was recorded as indicative of learning.

\section{Retention test}

Five days after the last day of conditioning, mice were submitted to a retention test consisting in four trials of $2 \mathrm{~min}$ in the same conditions as during conditioning (inter trial interval of $15 \mathrm{~min}$ and presence of the reward in the odorized hole).

\section{Data analysis}

For each training session, correct choices were averaged within groups. Between groups comparisons were done using ANOVA for repeated measures and Student $t$-tests for pair's comparisons. Statistical significance was set at $p<0.05$.

\section{SACRIFICE}

One hour after the last behavioral trial, the mice were deeply anesthetized (Pentobarbital, $0.2 \mathrm{~mL} / 30 \mathrm{~g}$ ) and killed by intracardiac perfusion of $50 \mathrm{ml}$ of fixative (4\% paraformaldehyde in phosphate buffer, $\mathrm{pH}$ 7.4). Brains were removed, post-fixed, cryoprotected in sucrose $(20 \%)$, frozen rapidly and then stored at $-20^{\circ} \mathrm{C}$ before sectioning with a cryostat (Jung).

\section{5-BROMO-2'-DEOXYURIDINE (BrdU) ADMINISTRATION}

BrdU (Sigma, $50 \mathrm{mg} / \mathrm{kg}$ in saline, three times at $2 \mathrm{~h}$ intervals) was injected 13 days before the behavioral training began in order to label a cohort of newborn cells arriving in the $\mathrm{OB}$ at the time of conditioning. 


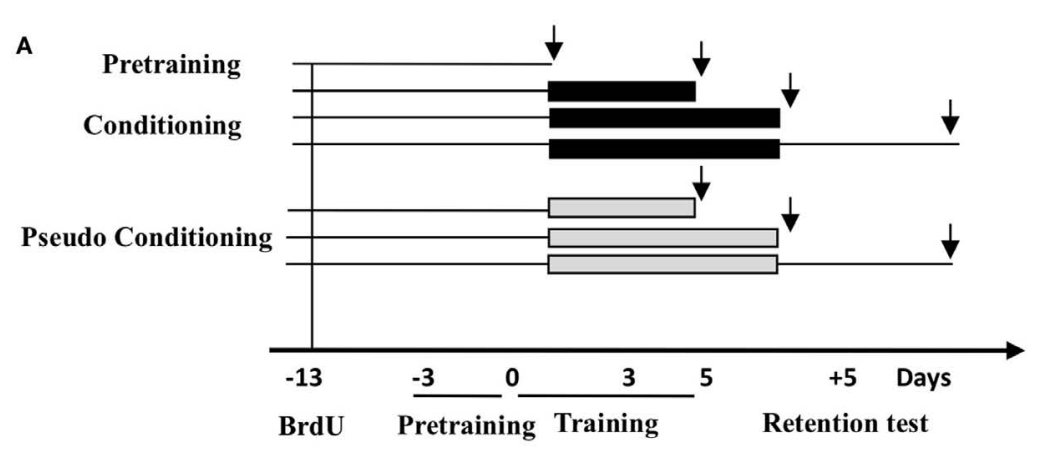

$\mathrm{Bi}$

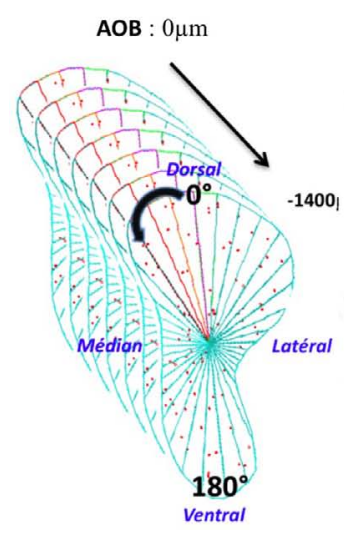

Bii

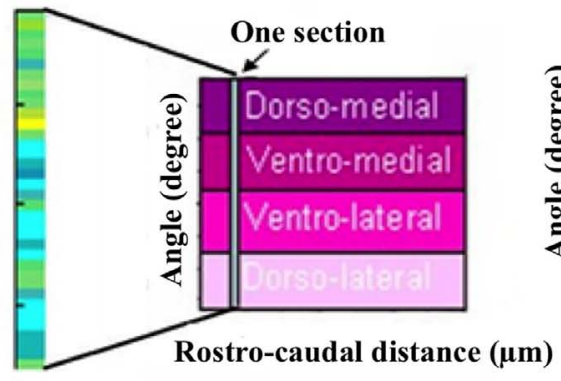

Biii

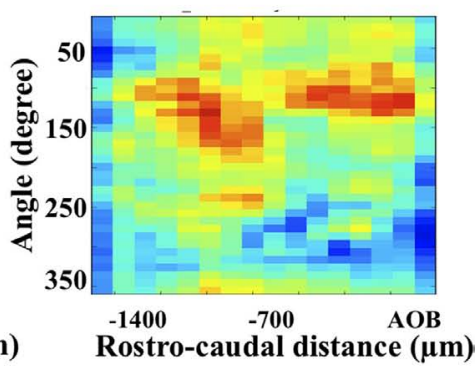

FIGURE 1 | Experimental design and cell mapping procedure. (A) Groups and timing of the experiment. Following a 3-day pre-training period, animals were submitted to an olfactory conditioning or pseudo-conditioning. They were sacrificed either before conditioning (pre-training) during (on day 3 and on day 5 of conditioning) or 5 days post conditioning, after a retention test. Black arrows indicate times of sacrifice, intervening $1 \mathrm{~h}$ after the last behavioral trial. To assess neurogenesis at these different time points, BrdU was administered 13 days before conditioning. (B) Principle of the labeled cell mapping method. Serial coronal sections of the OB were processed for BrdU or activated-caspase3 immunohistochemistry and every labeled cell was then counted on each section. The GCL was divided into 36 sectors of $10^{\circ}$ and a density of labeled profiles was calculated for each sector (Bi). The value of cell density obtained was then reported into a matrix in which one column represented all the sectors of one section. Sections were aligned along the rostro-caudal axis (Bii). Finally, pseudo-color representation of the matrix was generated, giving a 2-dimension image of the density of labeled cells in the GCL. An example of such representation is given (Biii).

\section{BrdU IMMUNOCYTOCHEMISTRY}

The protocol has been described previously (Mandairon et al., 2006a). Brains sections were first incubated in Target Retrieval Solution (Dako, Trappes, France) for $20 \mathrm{~min}$ at $98^{\circ} \mathrm{C}$. After cooling for $20 \mathrm{~min}$, they were treated with Triton $0.5 \%$ (SigmaX100) in phosphate buffered saline (PBS) for $30 \mathrm{~min}$, then for $3 \mathrm{~min}$ with pepsin $(0.43 \mathrm{U} / \mathrm{ml}$ in $0.1 \mathrm{~N} \mathrm{HCl}$, Sigma). Endogenous peroxidases were blocked with a solution of $3 \% \mathrm{H}_{2} \mathrm{O}_{2}$ in $0.1 \mathrm{M}$ PBS. Then, sections were incubated for $90 \mathrm{~min}$ in $5 \%$ normal horse serum, (Vector Laboratories, Burlingame, CA, USA) in 5\% bovine serum albumin, (BSA, Sigma) and $0.125 \%$ Triton X-100 to block non-specific binding, and then incubated overnight at $4^{\circ} \mathrm{C}$ in a mouse anti-BrdU primary antibody $(1 / 100$, Chemicon, Temecula, CA, USA). Sections were then incubated in a horse biotinylated anti-mouse secondary antibody (1/200, Vector) for $2 \mathrm{~h}$. Sections were then processed with avidin-biotin-peroxydase complex (ABC Elite Kit, Vector) for $30 \mathrm{~min}$. Finally, sections were reacted in $0.05 \%$ 3,3-diaminobenzidine-tetra-hydrochlo- ride (DAB, Sigma), $0.03 \% \mathrm{NiCL} 2$, and $0.03 \% \mathrm{H}_{2} \mathrm{O}_{2}$ in Tris- $\mathrm{HCl}$ buffer (0.05 M, pH 7.6), dehydrated in graded ethanols, and coverslipped in DPX.

\section{ACTIVATED CASPASE 3 IMMUNOHISTOCHEMISTRY}

We used the immunohistochemical detection of activatedcaspase 3 to detect apoptotic cell death. Brain sections were first rehydrated in PBS for $10 \mathrm{~min}$, then incubated in $\mathrm{H}_{2} \mathrm{O}_{2} 3 \%$ (prepared extemporaneously in PBS) for $20 \mathrm{~min}$. Sections were then treated with Triton 0.5\% (SigmaX100) BSA (2\%), Goat serum $(2 \%)$ in PBS for $1 \mathrm{~h}$. Then, sections were incubated for $1 \mathrm{~h}$ in 5\% normal goat serum, (Vector Laboratories, Burlingame, CA, USA) in $5 \%$ bovine serum albumin, (BSA, Sigma) and $0.125 \%$ Triton X-100 to block non-specific binding, and then incubated for $48 \mathrm{~h}$ at $4^{\circ} \mathrm{C}$ in a rabbit anti-cleaved (activated) caspase 3 primary antibody (1/500, asp175 \# 9661 Cell Signaling Technology). Sections were then incubated in a goat biotinylated anti-rabbit secondary antibody (1/200, Vector) for 1 h $30 \mathrm{~min}$. 
Sections were then processed with avidin-biotin-peroxydase complex (ABC Elite Kit, Vector) for $30 \mathrm{~min}$. Finally, sections were reacted in $0.05 \%$ 3,3-diaminobenzidine-tetra-hydrochlor ide (DAB,Sigma), $0.03 \% \mathrm{NiCL} 2$, and $0.03 \% \mathrm{H}_{2} \mathrm{O}_{2}$ in Tris- $\mathrm{HCl}$ buffer $(0.05 \mathrm{M}, \mathrm{pH} 7.6)$, dehydrated in graded ethanols, and coverslipped in DPX.

\section{QUANTIFICATION OF BrdU-POSITIVE CELLS AND ACTIVATED CASPASE 3-POSITIVE CELLS}

All cell counts were conducted blind with regards to the mouse status. Data were collected with the help of mapping software (Mercator Pro, Explora Nova, La Rochelle, France), coupled to a Zeiss microscope. In the GCL of the OB, every BrdU-positive cell was counted on 20 sections ( $14 \mu \mathrm{m}$ thick, $70 \mu \mathrm{m}$ intervals) of four to five mice per group. The number of positive cells was divided by the surface of the region of interest to yield the total density of labeled cells (number of labeled profiles $/ \mu \mathrm{m}^{2}$ ).

The same was done for activated-caspase3-positive cells. A recent study suggested that caspase 3 is not only a key mediator of apoptosis but could also function as a regulatory molecule in synaptic plasticity (D'Amelio et al., 2009). In our study, activated-caspase3-positive cells were counted only if the nucleus was stained. This enabled us to consider them as apoptotic cells and not as neurons undergoing a process of synaptic plasticity. Data were analyzed by ANOVA followed by Student $t$-tests for pair's comparisons.

\section{MAPPING OF BrdU-POSITIVE CELLS AND ACTIVATED CASPASE 3-POSITIVE CELLS IN THE GCL}

This was done according to a previously described procedure (Mandairon et al., 2006a, 2008; Busto et al., 2009; Sultan et al., 2010). The GCL was divided into 36 sectors of $10^{\circ}$ with a reference axis drawn parallel to the most ventral aspect of the subependymal layer of the OB (Figure 1Bi). The cell density (number of labeled profiles $/ \mu \mathrm{m}^{2}$ ) was calculated for each sector. Density values were then merged into arrays of $10^{\circ} \times 70 \mu \mathrm{m}$ bins in which one column represents one section (Figure 1Bii). The most rostral aspect of the accessory $\mathrm{OB}$ served as an anatomical landmark to align the sections across animals. For each sector, means across animals were calculated to yield a map of mean cell density. A colored image plot of the data was constructed in Matlab v.6 (Figure 1Biii). SD maps were also constructed. Using Matlab, the $20 \%$ highest density bins were selected in BrdU-positive cell maps as well as the $20 \%$ lowest density bins in the activatedcaspase3-positive cell maps. The overlapping bins were represented and counted. The percentage of overlapping bins (number of overlapping bins/number of bins selected $\times 100$ ) was calculated and compared between groups using a $t$-test for comparisons of proportion (Mandairon et al., 2006a).

\section{BrdU/NeuN DOUBLE LABELING AND ANALYSIS}

To determine the phenotype of BrdU-positive cells in the GCL of the $\mathrm{OB}$, we performed a BrdU/NeuN double-labeling using a rat anti-BrdU (1:100, Harlan Sera lab, Loughborough, UK) and a mouse anti-NeuN (1:500, Chemicon). Double-labelled cells were observed and analyzed by pseudo-confocal scanning microscopy using a Zeiss microscope equipped with the Apotome $(n=3-5$ animals per group, 30-40 cells per animal). A percentage of doublelabeled cells was calculated for each group and compared ANOVA and Student $t$-tests for pair's comparisons.

\section{ACTIVATED-CASPASE3/BrdU DOUBLE LABELING AND ANALYSIS}

To determine newborn cells death in the GCL, an activated caspase 3/BrdU double labeling was performed using a mouse anti-BrdU (1:100, Chemicon) and a rabbit anti anti-Activated caspase 3 (1:500, Cell signaling Technology) for $48 \mathrm{~h}$ at $4^{\circ} \mathrm{C}$. Then, sections were incubated $2 \mathrm{~h}$ at room temperature in secondary antibodies (goat anti-mouse Alexa 546-coupled, 1:200, Molecular Probes, Invitrogen Oregon USA and goat biotinylated anti-rabbit, 1/200, Vector) followed by Streptavidin Alexa 488 (1:1000, Molecular Probe) for $90 \mathrm{~min}$.

For data analysis, activated capspase3-positive cells were observed in each group ( $n=3$ animals per group, 8-10 cells per animal) and a percentage of BrdU-positive cells among activated-caspase3-positive cells was calculated for each group. Data were compared by ANOVA and Student $t$-tests for pair's comparisons.

\section{RESULTS}

\section{TEMPORAL RELATIONSHIP BETWEEN LEARNING AND NEWBORN NEURON SURVIVAL}

The global analysis of the behavioral data indicated that conditioned and pseudo-conditioned groups differed significantly [Group effect $F(1,20)=7.95, p<0.05$; Figure 2A]. The success rate increased in conditioned animals [Day effect $F(4,44)=4.99$, $p<0.05$ ] but not in pseudo-conditioned animals [Day effect $F(4,36)=0.29, p>0.05]$, indicating that only the conditioned animals learnt the association between the odor and the reward. Success rates in conditioned animals significantly differed from those of pseudo-conditioned animals on day 5 of learning and 5 days post learning ( $p<0.05$ in both cases; Figure $2 \mathrm{~A}$ ).

In animals randomly selected before (pre-training), during (day 3 and 5) or after (day +5$)$ conditioning or pseudo-conditioning, we counted the density of BrdU-positive cells (Figures 2B,D) in the GCL. The density of adult born cells evolved with conditioning or pseudo-conditioning according to a different pattern [Group effect $F(6,23)=3.53, p<0.05]$. In conditioned animals, the density of newborn cells was stable and similar to the pre-training level at all time points studied. Indeed, no difference was found between conditioned groups across time. In contrast, in pseudo-conditioned animals, newborn cell density decreased on day 5 and 5 days post training, compared to day 3 ( $p<0.05$; Figure $2 B$ ). Consequently, newborn cell density was significantly lower in pseudo-conditioned than in conditioned animals on day 5 of training and 5 days post training $(p<0.05)$. It is worth noting that the difference between conditioned and pseudo-conditioned animals with regard to newborn cell density appeared on day 5 of training, when the animals master the task. In contrast, on day 3 of training, at a time when the conditioned animals do not yet succeed in the task, the density of newborn cells was similar in conditioned and pseudo-conditioned groups. We also analyzed the level of neuronal differentiation of adult born cells across training using co-labeling of BrdU with the expression of the marker of mature neurons NeuN (Figures 2C,E). We found that the percentage of adult born neurons increased 


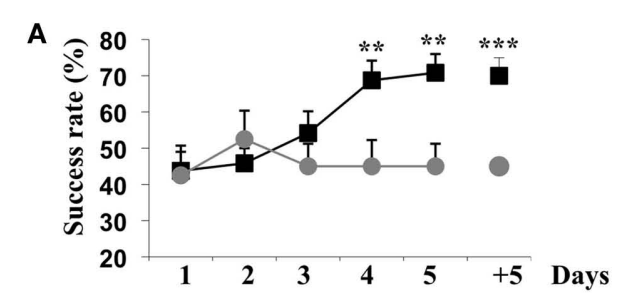

B

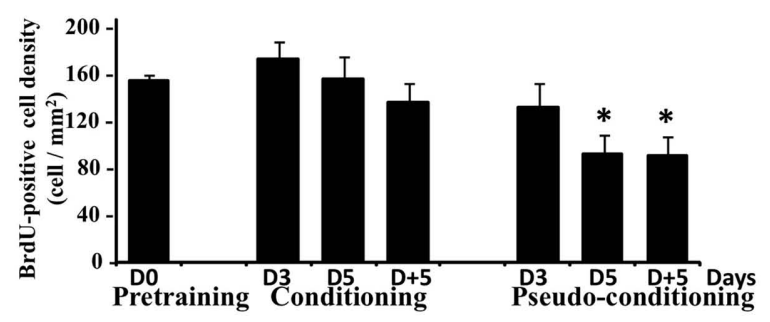

C

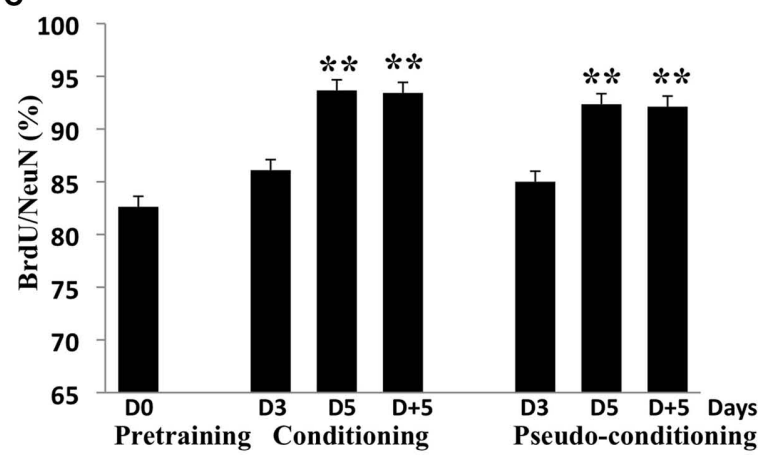

D

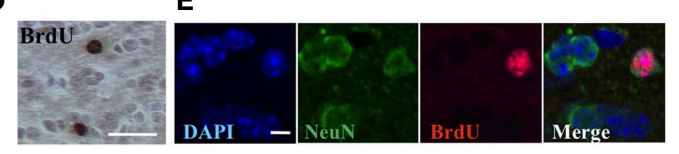

FIGURE 2 | Behavioral data and assessment of neurogenesis. (A) Success rate in conditioned and pseudo-conditioned animals during olfactory conditioning (day 1 to day 5 ) and in the retention test (day +5$) .{ }^{* *} p<0.005$ ${ }^{* * *} p<0.0005$ for difference between conditioned and pseudo-conditioned groups. (B) Density in BrdU-positive cells in the GCL before (pre-training) during (D3 and D5) and after ( $D+5$ ) olfactory conditioning or pseudoconditioning. ${ }^{*} p<0.05$ for difference from all other groups. (C) Percentage of BrdU-positive cells co-labeled with the neuronal marker NeuN in all experimental groups. ${ }^{* *} p<0.005$ for difference from pre-training level and from D3 in conditioned and pseudo-conditioned groups. (D) BrdU-positive cells in the GCL, bar $=15 \mu \mathrm{m}$. (E) BrdU/NeuN double-labeled cell in the GCL. DAPI stains all nuclei. Bar $=5 \mu \mathrm{m}$.

significantly from about $80 \%$ to $90 \%$ between day 3 and day 5 of training [Group effect $F(6,23)=4.32, p<0.005, p<0.05$ for difference between D3 and D5 in conditioned and pseudo-conditioned groups], and then remained stable between day 5 and 5 days post training. No difference was found between pre-training level and day 3 of training in any of the conditioned and pseudo-conditioned groups. These data indicated that the neuronal differentiation of newborn cells was completed 18-day post BrdU injections (13 days between BrdU injections and beginning of behavioral training +5 days of training) and that this temporal pattern of differentiation was independent of learning.
All together, these results indicated that the increased newborn cell density observed at the end of conditioning was due to the rescue from death of newborn cells occurring once behavioral performances raised meaning when the animals master the task (Figures 2A,B). This prompted us to examine whether cell death differentially affected newborn cells in the different experimental groups.

\section{NEWBORN NEURONS WERE SPARED BY CELL DEATH UPON ACQUISITION OF THE TASK}

In order to further document the death of newborn cells in pseudoconditioned animals and their rescue in conditioned animals, we performed activated-caspase $3 / \mathrm{BrdU}$ double-labeling experiment in which we looked at the percentage of BrdU-positive cells among those expressing activated-caspase 3 in the different experimental groups (Figures 3A,B). Results clearly showed that the percentage of newborn cells among dying cells decreased in conditioned animals on day 5 and 5 days post training, compared to the other groups [Group effect $F(6,14)=20.93, p<0.05$ ]. Furthermore, we counted activated-caspase3-positive cells in the GCL and found that their global density in the GCL was stable across time and groups [Group effect $F(6,25)=0.79, p>0.05$; Figures 3C,D]. Taken together, these results indicated that the process of learning a new olfactory task was accompanied by the selective rescue of BrdU-positive cells from apoptotic death to the detriment of non-labeled cells.

\section{SPATIO-TEMPORAL CORRELATION BETWEEN LOW CELL DEATH AND NEWBORN CELL SURVIVAL DURING LEARNING}

In a previous study, we showed that after learning, newborn cells were found at odor-specific locations in the GCL (Sultan et al., 2010). To assess the contribution of cell death to this regionalization of newborn cells, activated-caspase3-positive cells were mapped in the GCL and we compared this distribution to that of BrdU-positive cells. In all groups, a higher density of newborn cells was retrieved in the medial and anterior parts of the GCL (red areas), while the lowest density was observed in the latero-posterior area (yellow-green to blue areas) (Figure 4A, upper panel). This distribution can be explained by the presence of a medio-lateral and a antero-posterior gradients in global cell density which we described previously in the GCL (Busto et al., 2009). Thus, in all groups, the gross distribution of newborn cells was coherent with the cell density in the GCL (Figure 4A, upper panel). To assess the effect of conditioning or pseudo conditioning on the distribution of BrdU-positive cells, we calculated the ratio of density values in the pre-training group to those of all other groups and depicted the results as color-coded maps of the ratio values (Figure $\mathbf{4 A}$, lower panel). In conditioned animals, limited changes occurred in the lateral part of the GCL, accounting for the non-significant decrease in BrdU-positive cell density reported on Figure 2B. In contrast, in pseudo-conditioned animals, the ratio maps showed a loss of BrdU-positive cells, in line with the significant decreased in BrdU-cell density described in Figure $\mathbf{2 B}$, and that this loss occurred broadly in the GCL.

The mapping of activated-caspase3-positive cells also revealed a non-uniform distribution of cell death (Figure 4B, upper panel). More specifically, in conditioned animals, visual inspection of the activated- 


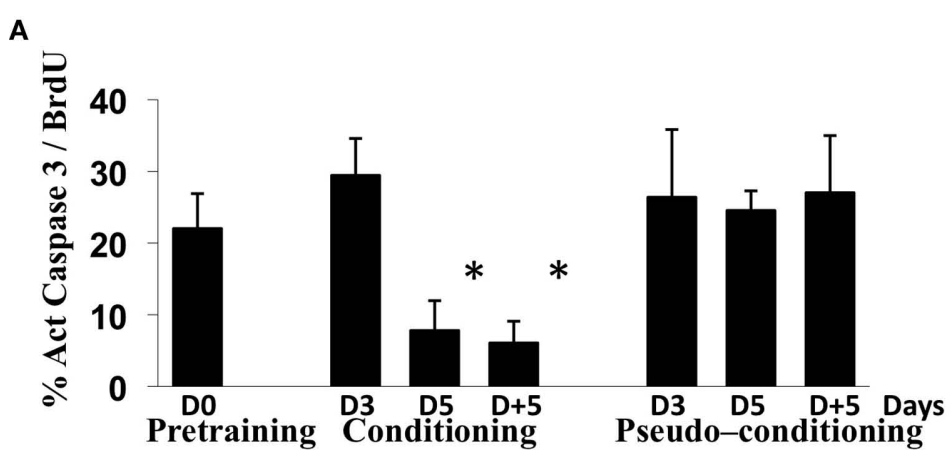

B
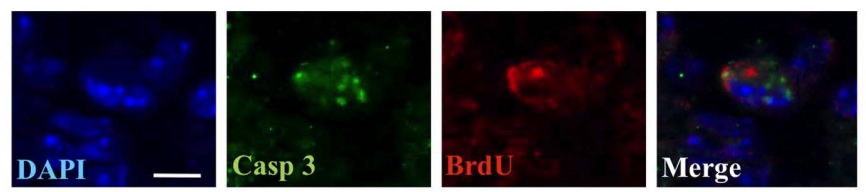

C

D

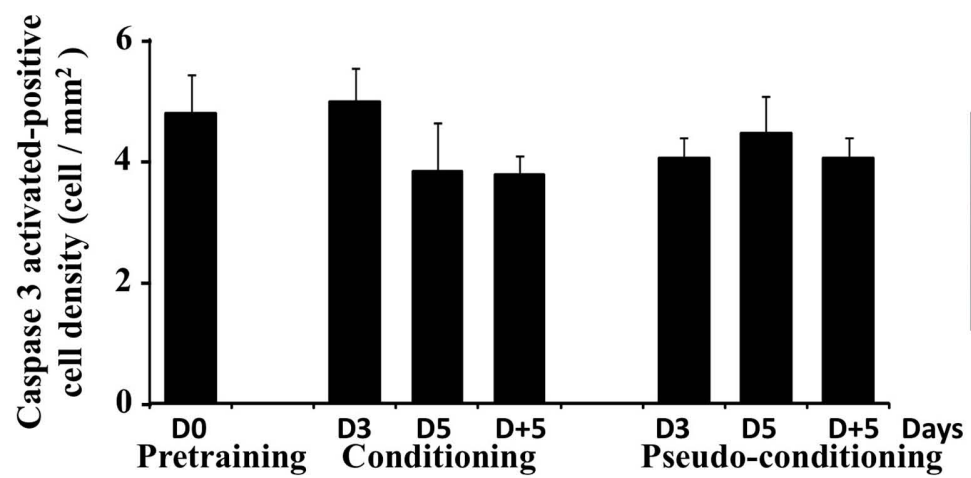

FIGURE 3 | Analysis of cell death during olfactory conditioning.

(A) Percentage of activated-caspase3/BrdU double-labeled cells in the GCL.

${ }^{*} p<0.05$ for difference from all other groups. (B) Activated-caspase3/BrdU double-labeled cell in the GCL. Bar $=5 \mu \mathrm{m}$. (C) Activated-caspase3-positive cell density in the GCL in all experimental groups. No significant difference was found. (D) Activated-caspase3-positive cells in the GCL. Bar $=15 \mu \mathrm{m}$. caspase3-positive cell density indicated that it was lower in the medial part of the GCL (dark blue areas), where BrdU-positive cell density was the highest. Cell death was thus lower in the medial part of the GCL than what could be expected if it was simply proportional to global or newborn cell density. In order to directly relate newborn cell survival to lower level of cell death, we compared for each group the locations of high BrdU-positive cell density with those of low cell death density and quantify the overlap between these regions (see Materials and Methods; Figure 4B, lower panel). Interestingly, we found that the overlap between areas of high newborn and low cell death densities was located mostly to the medial part of the GCL, and was significantly higher in conditioned groups compared to pre-training or pseudoconditioned groups (Figure 4C). Both BrdU and activated-caspase3 maps were highly reproducible within groups as illustrated by the SD maps, representing for each group, the SD value of the individual bins of the maps (Figure 4D). Taken together, these findings expanded the results of double-labeling experiment by suggesting that cell death was down-regulated in the areas of high density of BrdU-positive cells. However, on day 3 of conditioning, we found a high overlap of high
BrdU- and low activated-caspase3-positive cell densities despite the fact that double labeling indicated that the proportion of newborn cells among dying cells was not yet decreased suggesting that, at this time point, newborn neurons were not spared more than preexisting cells.

In summary, our findings indicate that in close temporal correlation with the rise in success rate in the task, a down-regulation of cell death was targeted to newborn neurons on D5 and D + 5, allowing their rescue at specific locations of the GCL.

\section{DISCUSSION}

While the increase in newborn neurons following associative olfactory learning has been well documented (Alonso et al., 2006; Kermen et al., 2010; Sultan et al., 2010), the issue of when and how are newborn neurons selected to survive has not been investigated. In this study, using olfactory conditioning combined to doublelabeling experiments and mapping of newborn or dying cells, we were able to show that cell death is regulated in the GCL of the OB during learning, allowing the learning-dependent rescue of newborn neurons. This conclusion is supported by the low pro- 

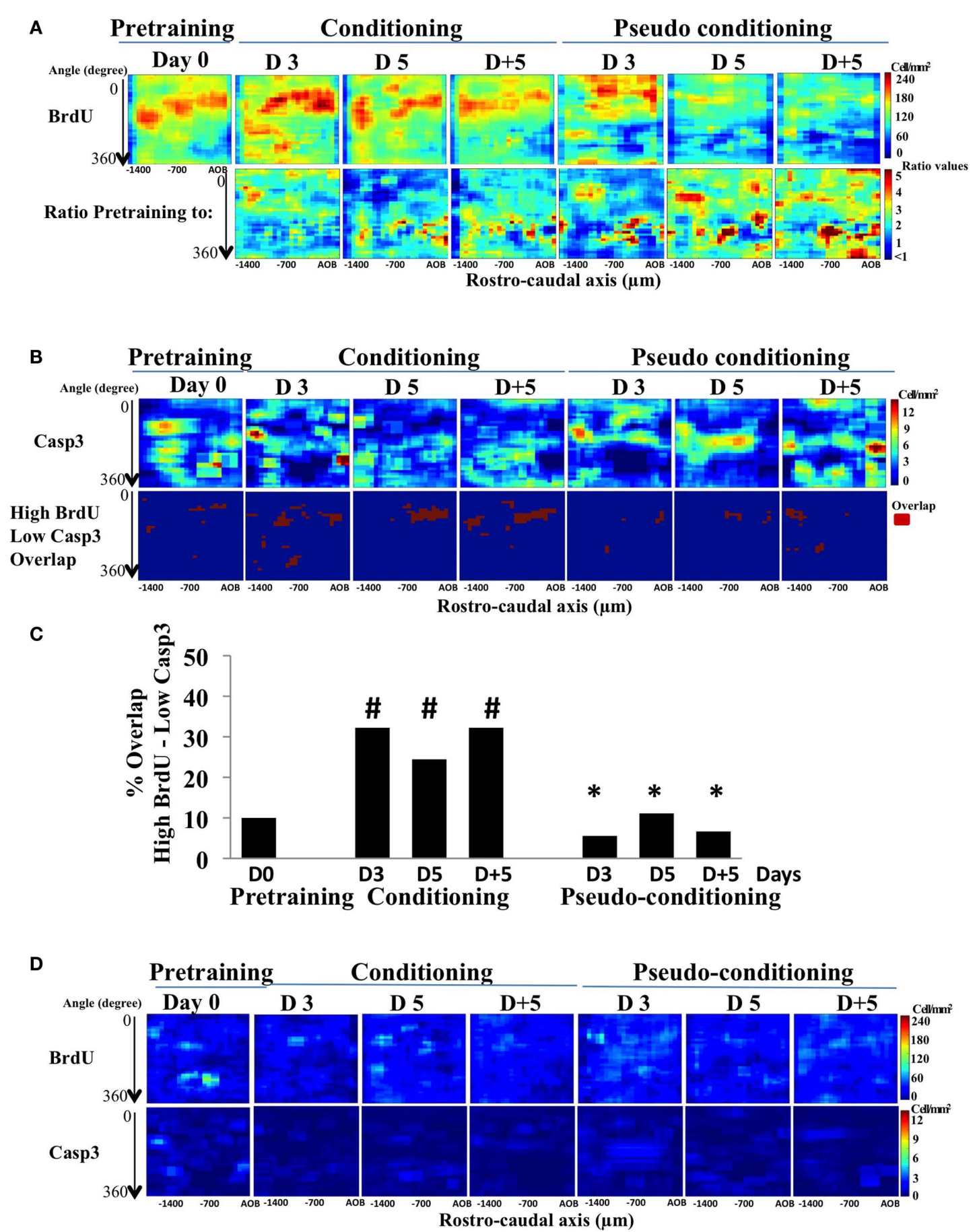

FIGURE 4 | BrdU- and activated-caspase3-positive cell mapping in the GCL. (A) Pseudo-color maps of Brdu-positive cells (BrdU; upper panel) and of the ratio BrdU-positive cell-density map of pre-training to all other groups (lower panel). (B) Activated-caspase3-positive (casp3) cell-density maps and maps of the overlap between high BrdU and low caspase 3 cell densities.
(C) Quantification of the overlap between high BrdU and low casp3. \#p<0.05 for difference from pre-training; ${ }^{*} p<0.05$ for difference from corresponding time points in conditioned groups. (D) Maps of standard deviation (SD maps) associated with each bin of the BrdU- and activated-caspase3-postiive cell maps shown in (A) portion of newborn cells undergoing cell death during learning and by the temporal coincidence we found between the increase in newborn neuron survival and the decrease of the death of newborn cells. Several features of this regulatory process are of particular interest. First, we showed that it depended on learning since it occurred only in conditioned animals and not in pseudoconditioned. Furthermore, it was closely correlated with the turning point of the learning curve. Indeed, it was only when animals 
mastered the task (day 5 of learning) that newborn neurons death decreased suggesting that having learnt the task may protect newborn neurons from death. This is consistent with our previous study, in which we showed that day-to-day memory consolidation processes were necessary for newborn neuron survival (Kermen et al., 2010). Memory consolidation processes and associated signaling (Izquierdo et al., 2006) could contribute to protect newborn neurons from death. A good candidate for linking memory consolidation and survival is BDNF whose expression is involved in memory formation (Cunha et al., 2010) and which has been shown to stimulate newborn neurons formation (Zigova et al., 1998; Benraiss et al., 2001; Pencea et al., 2001; Bath et al., 2008; but see also, Galvao et al., 2008).

Second, in our experiment, we also showed that the overall level of cell death was unaffected across experimental groups. This observation together with the decreased in the death BrdU-labeled cells observed on D5 and D + 5, indicated that BrdU-negative GCs were subjected to higher level of cell death. Some uncertainty remains with regards to the age of these GCs in which cell death increased upon learning. They could be either older or younger than the labeled cells (more or less than 13 days old at the onset of training). Previous works showing that newborn neurons aged more than 30 days are suppressed by learning to the benefit of younger neurons (Mandairon et al., 2006a; Mouret et al., 2008) strongly suggest that the target of increased death are likely to be pre existing GCs. However, it can not be excluded that younger GCs may also undergo cell death.

Finally, thanks to our mapping approach, we found that cell death was lower in the areas where a high BrdU-positive cell density was retrieved during and after learning. These results support the idea of a regionalization of cell death. Interestingly, the target of cell death, newborn cells or pre-exisiting cells is likely to evolve with learning. Indeed, a mismatch was revealed by our study regarding cell death at 3 days of conditioning. At this time point, we found a high overlap between regions of high BrdU- and low activated-caspase3-positive cell densities but activated-caspase3/BrdU double labeling revealed no decrease in the proportion of newborn neurons among dying

\section{REFERENCES}

Alonso, M., Viollet, C., Gabellec, M. M., Meas-Yedid, V., Olivo-Marin, J. C., and Lledo, P. M. (2006). Olfactory discrimination learning increases the survival of adult-born neurons in the olfactory bulb. J. Neurosci. 26, 10508-10513.

Bath, K. G., Mandairon, N., Jing, D., Rajagopal, R., Kapoor, R., Chen, Z. Y., Khan, T., Proenca, C. C., Kraemer, R., Cleland, T.A., Hempstead, B. L., Chao, M. V., and Lee, F. S. (2008). Variant brain-derived neurotrophic factor (Val66Met) alters adult olfactory bulb neurogenesis and spontaneous olfactory discrimination. J. Neurosci. 28, 2383-2393.

Benraiss, A., Chmielnicki, E., Lerner, K., Roh, D., and Goldman, S. A. (2001).
Adenoviral brain-derived neurotrophic factor induces both neostriatal and olfactory neuronal recruitment from endogenous progenitor cells in the adult forebrain. J. Neurosci. 21, 6718-6731.

Bovetti, S., Veyrac, A., Peretto, P., Fasolo, A., and De Marchis, S. (2009). Olfactory enrichment influences adult neurogenesis modulating GAD67 and plasticity-related molecules expression in newborn cells of the olfactory bulb. PLoS ONE4, e6359. doi: 10.1371/journal.pone.0006359

Busto, G. U., Elie, J. E., Kermen, F., Garcia, S., Sacquet, J., Jourdan, F., Marcel, D., Mandairon, N., and Didier, A. (2009). Expression of Zif268 in the granule cell layer of the adult mouse olfactory bulb is modulated by experience. Eur. J. Neurosci. 29, 1431-1439.

cells. It could thus be proposed that learning regulates cell death in two steps according to the learning process: a first early step with a down regulation of cell death occurring in high BrdUpositive cell density areas, without sparing newborn cells and a second step that could depend on the rise of performances and the strength of memory during which down regulation of cell death would target more specifically newborn neurons in these selected areas. Support to specific mechanisms occurring in newborn neurons and not older neurons upon memory formation is brought by the demonstration that newborn neurons selectively express long-term potentiation in the OB (Nissant et al., 2009).

The regulation of cell death we reported here thus shapes the temporal and spatial patterns of learning-dependent newborn neurons survival. These newborn neurons will be later on integrated in the network supporting long-term memory (Lazarini et al., 2009; Sultan et al., 2010). Based on these findings, one may predict that blocking cell death would alter the turn over of GCs and affect long-term memory. However, a recent study reported no effect of a pharmacological blockade by the pancaspase inhibitor Z-Vad (Mouret et al., 2009) on olfactory long-term memory. A possible explanation is that Z-Vad treatment decreased the overall level of cell death but did not prevent a learning-dependent protection of newborn neurons. Indeed, in this study conditioned animals displayed a lower level of cell death than pseudo-conditioned animals. This would imply that the absolute level of survival is less critical for memory than learning-dependent cell death regulation of newborn neurons.

In conclusion, this study demonstrates that newborn neurons are rescued from apoptotic death according to a specific time course closely following that of learning the associative task, through a process of regulation of cell death targeted to newborn cells as soon as the task is mastered.

\section{ACKNOWLEDGMENTS}

This work was supported by CNRS and University Claude Bernard Lyon 1 and the French ministry of research (Fellowship to Sébastien Sultan).

Cunha, C., Brambilla, R., and Thomas, K. L. (2010). A simple role for BDNF in learning and memory? Front. Mol. Neurosci. 3:1. doi: 10.3389/ neuro.02.001.2010

D'Amelio, M., Cavallucci, V., and Cecconi, F. (2009). Neuronal caspase-3 signaling: not only cell death. Cell Death Differ. 17, 1104-1114.

Galvao, R. P., Garcia-Verdugo, J. M., and Alvarez-Buylla, A. (2008). Brainderived neurotrophic factor signaling does not stimulate subventricular zone neurogenesis in adult mice and rats. J. Neurosci. 28, 13368-13383.

Izquierdo, I., Bevilaqua, L. R., Rossato, J. I., Bonini, J. S., Medina, J. H., and Cammarota, M. (2006). Different molecular cascades in different sites of the brain control memory consolidation. Trends Neurosci. 29, 496-505.

Kay, L. M., and Laurent, G. (1999). Odorand context-dependent modulation of mitral cell activity in behaving rats. Nat. Neurosci. 2, 1003-1009.

Kelsch, W., Lin, C. W., Mosley, C. P., and Lois, C. (2009). A critical period for activity-dependent synaptic development during olfactory bulb adult neurogenesis. J. Neurosci. 29, 11852-11858.

Kermen, F., Sultan, S., Sacquet, J., Mandairon, N., and Didier, A. (2010). Consolidation of an olfactory memory trace in the olfactory bulb is required for learning-induced survival of adultborn neurons and long-term memory. PLoS ONE 5, e12118. doi: 10.1371/ journal.pone.0012118 
Lazarini, F., Mouthon, M. A., Gheusi, G., de Chaumont, F., Olivo-Marin, J. C., Lamarque, S., Abrous, D. N., Boussin, F. D., and Lledo, P. M. (2009). Cellular and behavioral effects of cranial irradiation of the subventricular zone in adult mice. PLoS ONE 4, e7017. doi: 10.1371/journal.pone.0007017

Livneh, Y., Feinstein, N., Klein, M., and Mizrahi, A. (2009). Sensory input enhances synaptogenesis of adultborn neurons. J. Neurosci. 29, 86-97.

Lledo, P. M., Alonso, M., and Grubb, M.S. (2006). Adult neurogenesis and functional plasticity in neuronal circuits. Nat. Rev. Neurosci. 7, 179-193.

Mandairon, N., Didier, A., and Linster, C. (2008). Odor enrichment increases interneurons responsiveness in spatially defined regions of the olfactory bulb correlated with perception. Neurobiol. Learn. Mem. 90, 178-184.

Mandairon, N., Sacquet, J., Garcia, S., Ravel, N., Jourdan, F., and Didier, A. (2006a). Neurogenic correlates of an olfactory discrimination task in the adult olfactory bulb. Eur. J. Neurosci. 24, 3578-3588.

Mandairon, N., Sacquet, J., Jourdan, F., and Didier, A. (2006b). Long-term fate and distribution of newborn cells in the adult mouse olfactory bulb: influences of olfactory deprivation. Neuroscience 141, 443-451.

Mandairon, N., Sultan, S., Rey, N., Kermen, F., Moreno, M., Busto, G., Farget, V., Messaoudi, B., Thevenet, M., and Didier, A. (2009). A computer-assisted odorized hole-board for testing olfactory perception in mice. J. Neurosci. Methods 180, 296-303.

Martin, C., Gervais, R., Hugues, E., Messaoudi, B., and Ravel, N. (2004).
Learning modulation of odor-induced oscillatory responses in the rat olfactory bulb: a correlate of odor recognition? J. Neurosci. 24, 389-397.

Moreno, M. M., Linster, C., Escanilla, O., Sacquet, J., Didier, A., and Mandairon, N. (2009). Olfactory perceptual learning requires adult neurogenesis. Proc. Natl. Acad. Sci. U.S.A. 106, 17980-17985.

Mouret, A., Gheusi, G., Gabellec, M. M., de Chaumont, F., Olivo-Marin, J. C., and Lledo, P. M. (2008). Learning and survival of newly generated neurons: when time matters. J. Neurosci. 28, 11511-11516.

Mouret, A., Lepousez, G., Gras, J., Gabellec, M. M., and Lledo, P. M. (2009). Turnover of newborn olfactory bulb neurons optimizes olfaction. J. Neurosci. 29, 12302-12314.

Nissant,A., Bardy, C., Katagiri, H., Murray, K., and Lledo, P. M. (2009). Adult neurogenesis promotes synaptic plasticity in the olfactory bulb. Nat. Neurosci. 12, 728-730.

Pencea, V., Bingaman, K. D., Wiegand, S. J., and Luskin, M. B. (2001). Infusion of brain-derived neurotrophic factor into the lateral ventricle of the adult rat leads to new neurons in the parenchyma of the striatum, septum, thalamus, and hypothalamus. J. Neurosci. 21, 6706-6717.

Petreanu, L., and Alvarez-Buylla, A. (2002). Maturation and death of adult-born olfactory bulb granule neurons: role of olfaction. J. Neurosci. 22, 6106-6113.

Rochefort, C., Gheusi, G., Vincent, J. D., and Lledo, P. M. (2002). Enriched odor exposure increases the number of newborn neurons in the adult olfac- tory bulb and improves odor memory. J. Neurosci. 22, 2679-2689.

Salcedo, E., Zhang, C., Kronberg, E., and Restrepo, D. (2005). Analysis of training-induced changes in ethyl acetate odor maps using a new computational tool to map the glomerular layer of the olfactory bulb. Chem. Senses 30 615-626.

Shepherd, G. M., Chen, W. R., Willhite, D. Migliore, M., and Greer, C. A. (2007). The olfactory granule cell: from classical enigma to central role in olfactory processing. Brain Res. Rev. 55 , 373-382.

Sultan, S., Mandairon, N., Kermen, F., Garcia, S., Sacquet, J., and Didier, A (2010). Learning-dependent neurogenesis in the olfactory bulb determines long-term olfactory memory. FASEB J. 24, 2355-2363.

Valley, M. T., Mullen, T. R., Schultz, L. C., Sagdullaev, B. T., and Firestein, S. (2009). Ablation of mouse adult neurogenesis alters olfactory bulb structure and olfactory fear conditioning. Front. Neurosci. 3:51. doi: 10.3389/ neuro.22.003.2009

Veyrac, A., Sacquet, J., Nguyen, V., Marien, M., Jourdan, F., and Didier, A. (2009). Novelty determines the effects of olfactory enrichment on memory and neurogenesis through noradrenergic mechanisms. Neuropsychopharmacology34,786-795.

Winner, B., Cooper-Kuhn, C. M., Aigner, R., Winkler, J., and Kuhn, H. G. (2002). Long-term survival and cell death of newly generated neurons in the adult rat olfactory bulb. Eur. J. Neurosci. 16, 1681-1689.

Yamaguchi, M., and Mori, K. (2005). Critical period for sensory expe- rience-dependent survival of newly generated granule cells in the adult mouse olfactory bulb. Proc. Natl. Acad. Sci. U.S.A. 102, 9697-9702.

Zigova, T., Pencea, V., Betarbet, R., Wiegand, S. J., Alexander, C., Bakay, R. A., and Luskin, M. B. (1998). Neuronal progenitor cells of the neonatal subventricular zone differentiate and disperse following transplantation into the adult rat striatum. Cell Transplant. 7, 137-156.

Conflict of Interest Statement: The authors declare that the research was conducted in the absence of any commercial or financial relationships that could be construed as a potential conflict of interest.

Received: 27 January 2011; accepted: 29 March 2011; published online: 03 May 2011.

Citation: Sultan S, Lefort JM, Sacquet J, Mandairon N and Didier A (2011) Acquisition of an olfactory associative task triggers a regionalized downregulation of adult born neuron cell death. Front. Neurosci. 5:52. doi: 10.3389/ fnins.2011.00052

This article was submitted to Frontiers in Neurogenesis, a specialty of Frontiers in Neuroscience.

Copyright (C) 2011 Sultan, Lefort, Sacquet, Mandairon and Didier. This is an openaccess article subject to a non-exclusive license between the authors and Frontiers Media SA, which permits use, distribution and reproduction in other forums, provided the original authors and source are credited and other Frontiers conditions are complied with. 